\title{
Low temperature relation for the trace of the energy-momentum tensor in QCD with light quarks
}

\author{
N.O.Agasian \\ State Research Center \\ Institute of Theoretical and Experimental Physics, \\ Moscow 117218, Russia
}

\begin{abstract}
It is shown that the temperature derivatives of the anomalous and normal (quark massive term) contributions to the trace of the energy-momentum tensor in QCD are equal to each other in the low temperature region. The physical consequences of this relation are discussed.
\end{abstract}

1. The low-energy theorems, playing an important role in the understanding of the vacuum state properties in quantum field theory, were discovered almost at the same time as quantum field methods have been applied in particle physics (see, for example, Low theorems [1]). In QCD, they were obtained in the beginning of eighties [2. The QCD low-energy theorems, being derived from the very general symmetry considerations and not depending on the details of confinement mechanism, sometimes give information which is not easy to obtain in another way. Also, they can be used as "physically sensible" restrictions in the constructing of effective theories. Recently, they were generalized to finite temperature and chemical potential case [3, 4 . These theorems were used for investigation of $\mathrm{QCD}$ vacuum phase structure in a magnetic field [5] and at finite temperature [6].

The investigation of the vacuum state behavior under the influence of various external factors is known to be one of the central problems of quantum field theory. In the realm of strong interactions (QCD) the main factors are the temperature and the baryon density. At low temperatures, $T<T_{c}\left(T_{c}\right.$-temperature of the "hadronquark-gluon" phase transition ), the dynamics of QCD is essentially nonperturbative and is characterized by confinement and spontaneous breaking of chiral symmetry (SBCS). In the hadronic phase the partition function of the system is dominated by the contribution of the lightest particles in the physical spectrum. It is well known that due to the smallness of pion mass as compared to the typical scale of strong interactions, the pion plays a special role among other strong-interacting particles. Therefore for many problems of QCD at zero temperature the chiral limit, $M_{\pi} \rightarrow 0$, is an appropriate one. On the other hand a new mass scale emerges in the physics of QCD phase transitions, namely the critical transition temperature $T_{c}$. Numerically the critical transition temperature turns out to be close to the pion mass, $T_{c} \approx M_{\pi}$ [7]. However hadron states heavier than pion have masses several times larger than $T_{c}$ and therefore their contribution to the thermodynamic quantities is damped by Boltzmann factor $\sim \exp \left\{-M_{\text {hadr }} / T\right\}$. Thus the thermodynamics of the low temperature hadron phase, $T \lesssim M_{\pi}$,

*Electronic address: agasian@heron.itep.ru is described basically in terms of the thermal excitations of relativistic massive pions.

In the present paper the low temperature relation for the trace of the energy-momentum tensor in QCD with two light quarks is obtained based on the general dimensional and renormalization-group properties of the QCD partition function and dominating role of the pion thermal exitations in the hadronic phase. The physical consequences of this relation are discussed as well as the possibilities to use it in the lattice studies of the QCD at finite temperature.

2 . For non-zero quark mass $\left(m_{q} \neq 0\right)$ the scale invariance is broken already at the classical level. Therefore the pion thermal excitations would change, even in the ideal gas approximation, the value of the gluon condensate with increasing temperature [8]. To determine this dependence use will be made of the general renormalization and scale properties of the QCD partition function. This is a standard method and it is used for derivation of low-energy QCD theorems [2-6]. In Ref. [9] this method was used for investigation of the thermodynamic properties of QCD nonperturbative vacuum with two flavors at low temperature outside of the scope of perturbation theory. In what follows we stick to the approach outlined in 9].

The QCD Euclidean partition function with two quark flavors has the following form $(\beta=1 / T)$

$$
Z=\int[D A] \prod_{q=u, d}[D \bar{q}][D q] \exp \left\{-\int_{0}^{\beta} d x_{4} \int_{V} d^{3} x \mathcal{L}\right\} .
$$

Here the QCD Lagrangian is

$$
\mathcal{L}=\frac{1}{4 g_{0}^{2}}\left(G_{\mu \nu}^{a}\right)^{2}+\sum_{q=u, d} \bar{q}\left[\gamma_{\mu}\left(\partial_{\mu}-i \frac{\lambda^{a}}{2} A_{\mu}^{a}\right)+m_{0 q}\right] q
$$

where the gauge fixing and ghost terms have been omitted. The free energy density is given by the relation $\beta V F$ $\left(T, m_{0 u}, m_{0 d}\right)=-\ln Z$. Eq. (11) yields the following expression for the gluon condensate $\left(\left\langle G^{2}\right\rangle \equiv\left\langle\left(G_{\mu \nu}^{a}\right)^{2}\right\rangle\right)$

$$
\left\langle G^{2}\right\rangle\left(T, m_{0 u}, m_{0 d}\right)=4 \frac{\partial F}{\partial\left(1 / g_{0}^{2}\right)} .
$$

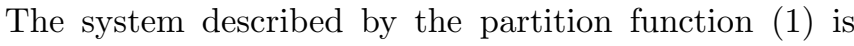
characterized by the set of dimensionful parameters 
$M, T, m_{0 q}(M)$ and dimensionless charge $g_{0}^{2}(M)$, where $M$ is the ultraviolet cutoff. On the other hand one can consider the renormalized free energy $F_{R}$ and by using the dimensional and renormalization-group properties of $F_{R}$ recast (3) into the form containing derivatives with respect to the physical parameter $T$ and renormalized masses $m_{q}$.

The phenomenon of dimensional transmutation results in the appearance of a nonperturbative dimensionful parameter

$$
\Lambda=M \exp \left\{\int_{\alpha_{s}(M)}^{\infty} \frac{d \alpha_{s}}{\beta\left(\alpha_{s}\right)}\right\},
$$

where $\alpha_{s}=g_{0}^{2} / 4 \pi$, and $\beta\left(\alpha_{s}\right)=d \alpha_{s}(M) / d \ln M$ is the Gell-Mann-Low function. Furthermore, as it is well known, the quark mass has anomalous dimension and depends on the scale $M$. The renormalization -group equation for $m_{0}(M)$, the running mass, is $d \ln m_{0} / d \ln M=$ $-\gamma_{m}$ and we use the $\overline{M S}$ scheme for which $\beta$ and $\gamma_{m}$ are independent of the quark mass [4, 10]. Upon integration the renormalization-group invariant mass is given by

$$
m_{q}=m_{o q}(M) \exp \left\{\int^{\alpha_{s}(M)} \frac{\gamma_{m_{q}}\left(\alpha_{s}\right)}{\beta\left(\alpha_{s}\right)} d \alpha_{s}\right\},
$$

where the indefinite integral is evaluated at $\alpha_{s}(M)$. Next we note that since free energy is renormalization-group invariant quantity its anomalous dimension is zero. Thus $F_{R}$ has only a normal (canonical) dimension equal to 4 . Making use of the renorm-invariance of $\Lambda$, one can write in the most general form

$$
F_{R}=\Lambda^{4} f\left(\frac{T}{\Lambda}, \frac{m_{u}}{\Lambda}, \frac{m_{d}}{\Lambda}\right),
$$

where $f$ is some function. From (伍), (5) and (6) one gets

$$
\begin{gathered}
\frac{\partial F_{R}}{\partial\left(1 / g_{0}^{2}\right)}=\frac{\partial F_{R}}{\partial \Lambda} \frac{\partial \Lambda}{\partial\left(1 / g_{0}^{2}\right)}+\sum_{q} \frac{\partial F_{R}}{\partial m_{q}} \frac{\partial m_{q}}{\partial\left(1 / g_{0}^{2}\right)}, \\
\frac{\partial m_{q}}{\partial\left(1 / g_{0}^{2}\right)}=-4 \pi \alpha_{s}^{2} m_{q} \frac{\gamma_{m_{q}}\left(\alpha_{s}\right)}{\beta\left(\alpha_{s}\right)} .
\end{gathered}
$$

With the account of (3) the gluon condensate is given by

$$
\begin{gathered}
\left\langle G^{2}\right\rangle\left(T, m_{u}, m_{d}\right) \\
=\frac{16 \pi \alpha_{s}^{2}}{\beta\left(\alpha_{s}\right)}\left(4-T \frac{\partial}{\partial T}-\sum_{q}\left(1+\gamma_{m_{q}}\right) m_{q} \frac{\partial}{\partial m_{q}}\right) F_{R} .
\end{gathered}
$$

It is convenient to choose such a large scale that one can take the lowest order expressions, $\beta\left(\alpha_{s}\right) \rightarrow-b \alpha_{s}^{2} / 2 \pi$, where $b=\left(11 N_{c}-2 N_{f}\right) / 3$ and $1+\gamma_{m} \rightarrow 1$. Thus, we have the following equations for condensates

$$
\left\langle G^{2}\right\rangle(T)=-\frac{32 \pi^{2}}{b}\left(4-T \frac{\partial}{\partial T}-\sum_{q} m_{q} \frac{\partial}{\partial m_{q}}\right) F_{R} \equiv-\hat{D} F_{R},
$$

$$
\langle\bar{q} q\rangle(T)=\frac{\partial F_{R}}{\partial m_{q}} .
$$

3. In the hadronic phase the effective pressure from which one can extract the condensates $\langle\bar{q} q\rangle(T)$ and $\left\langle G^{2}\right\rangle(T)$ using the general relations (10) and (11) has the form

$$
P_{e f f}(T)=-\varepsilon_{v a c}+P_{h}(T),
$$

where $\varepsilon_{v a c}=\frac{1}{4}\left\langle\theta_{\mu \mu}\right\rangle$ is the nonperturbative vacuum energy density at $T=0$ and

$$
\left\langle\theta_{\mu \mu}\right\rangle=-\frac{b}{32 \pi^{2}}\left\langle G^{2}\right\rangle+\sum_{q=u, d} m_{q}\langle\bar{q} q\rangle
$$

is the trace of the energy-momentum tensor. In Eq.(12) $P_{h}(T)$ is the thermal hadrons pressure. The quark and gluon condensates are given by the equations

$$
\begin{gathered}
\langle\bar{q} q\rangle(T)=-\frac{\partial P_{e f f}}{\partial m_{q}}, \\
\left\langle G^{2}\right\rangle(T)=\hat{D} P_{e f f},
\end{gathered}
$$

where the operator $\hat{D}$ is defined by the relation (10)

$$
\hat{D}=\frac{32 \pi^{2}}{b}\left(4-T \frac{\partial}{\partial T}-\sum_{q} m_{q} \frac{\partial}{\partial m_{q}}\right) .
$$

Consider the $T=0$ case. One can use the low energy theorem for the derivative of the gluon condensate with respect to the quark mass [2]

$$
\frac{\partial}{\partial m_{q}}\left\langle G^{2}\right\rangle=\int d^{4} x\left\langle G^{2}(0) \bar{q} q(x)\right\rangle=-\frac{96 \pi^{2}}{b}\langle\bar{q} q\rangle+O\left(m_{q}\right),
$$

where $O\left(m_{q}\right)$ stands for the terms linear in light quark masses. Then one arrives at the following relation [11]

$$
\frac{\partial \varepsilon_{v a c}}{\partial m_{q}}=-\frac{b}{128 \pi^{2}} \frac{\partial}{\partial m_{q}}\left\langle G^{2}\right\rangle+\frac{1}{4}\langle\bar{q} q\rangle=\frac{3}{4}\langle\bar{q} q\rangle+\frac{1}{4}\langle\bar{q} q\rangle=\langle\bar{q} q\rangle .
$$

Note that three fourths of the quark condensate stem from the gluon part of the nonperturbative vacuum energy density. Along the same lines one arrives at the expression for the gluon condensate

$$
-\hat{D} \varepsilon_{v a c}=\left\langle G^{2}\right\rangle \text {. }
$$

In order to get the dependence of the quark and gluon condensates upon $T$ use is made of the Gell-MannOakes-Renner (GMOR) relation $(\Sigma=|\langle\bar{u} u\rangle|=|\langle\bar{d} d\rangle|)$

$$
F_{\pi}^{2} M_{\pi}^{2}=-\frac{1}{2}\left(m_{u}+m_{d}\right)\langle\bar{u} u+\bar{d} d\rangle=\left(m_{u}+m_{d}\right) \Sigma
$$

Then we can find the following relations

$$
\frac{\partial}{\partial m_{q}}=\frac{\Sigma}{F_{\pi}^{2}} \frac{\partial}{\partial M_{\pi}^{2}},
$$




$$
\begin{gathered}
\sum_{q} m_{q} \frac{\partial}{\partial m_{q}}=\left(m_{u}+m_{d}\right) \frac{\Sigma}{F_{\pi}^{2}} \frac{\partial}{\partial M_{\pi}^{2}}=M_{\pi}^{2} \frac{\partial}{\partial M_{\pi}^{2}}, \\
\hat{D}=\frac{32 \pi^{2}}{b}\left(4-T \frac{\partial}{\partial T}-M_{\pi}^{2} \frac{\partial}{\partial M_{\pi}^{2}}\right) .
\end{gathered}
$$

Within the described above framework one can derive the thermodynamic relation for the quantum anomaly in the trace of the energy-momentum tensor of QCD. At low temperature the main contribution to the pressure comes from thermal excitations of massive pions. The general expression for the pressure reads

$$
P_{\pi}=T^{4} \varphi\left(M_{\pi} / T\right)
$$

where $\varphi$ is a function of the ratio $M_{\pi} / T$. Then the following relation is valid

$$
\left(4-T \frac{\partial}{\partial T}-M_{\pi}^{2} \frac{\partial}{\partial M_{\pi}^{2}}\right) P_{\pi}=M_{\pi}^{2} \frac{\partial P_{\pi}}{\partial M_{\pi}^{2}} .
$$

With the account of $(14,15), 18,22)$ and (25) one gets

$$
\Delta\langle\bar{q} q\rangle=-\frac{\partial P_{\pi}}{\partial m_{q}}, \quad \Delta\left\langle G^{2}\right\rangle=\frac{32 \pi^{2}}{b} M_{\pi}^{2} \frac{\partial P_{\pi}}{\partial M_{\pi}^{2}},
$$

where $\Delta\langle\bar{q} q\rangle=\langle\bar{q} q\rangle_{T}-\langle\bar{q} q\rangle$ and $\Delta\left\langle G^{2}\right\rangle=\left\langle G^{2}\right\rangle_{T}-\left\langle G^{2}\right\rangle$. In view of (22) one can recast (26) in the form

$$
\Delta\left\langle G^{2}\right\rangle=-\frac{32 \pi^{2}}{b} \sum_{q} m_{q} \Delta\langle\bar{q} q\rangle
$$

Let us divide both sides of (27) by $\Delta T$ and take the limit $\Delta T \rightarrow 0$. This yields

$$
\frac{\partial\left\langle G^{2}\right\rangle}{\partial T}=-\frac{32 \pi^{2}}{b} \sum_{q} m_{q} \frac{\partial\langle\bar{q} q\rangle}{\partial T} .
$$

This can be rewritten as

$$
\frac{\partial\left\langle\theta_{\mu \mu}^{g}\right\rangle}{\partial T}=\frac{\partial\left\langle\theta_{\mu \mu}^{q}\right\rangle}{\partial T}
$$

where $\left\langle\theta_{\mu \mu}^{q}\right\rangle=\sum m_{q}\langle\bar{q} q\rangle$ and $\left\langle\theta_{\mu \mu}^{g}\right\rangle=$ $\left(\beta\left(\alpha_{s}\right) / 16 \pi \alpha_{s}^{2}\right)\left\langle G^{2}\right\rangle$ are correspondingly the quark and gluon contributions to the trace of the energymomentum tensor. Note that in deriving this result use was made of the low energy GMOR relation, and therefore the thermodynamic relation $(28,29)$ is valid in the light quark theory.

In Ref. [9] it was shown that dilute gas approximation for the relativistic massive pions is valid at temperature $T \lesssim M_{\pi}$. The pressure of the massive relativistic pion gas has the form

$$
P_{\pi}(T)=\frac{3 M_{\pi}^{2} T^{2}}{2 \pi^{2}} \sum_{n=1}^{\infty} \frac{1}{n^{2}} K_{2}\left(n \frac{M_{\pi}}{T}\right),
$$

where $K_{2}$ is the Mackdonald function. Making use of the relations (14-23) and pressure (30) we find the following expressions for the condensates $\Sigma(T)$ and $\left\langle G^{2}\right\rangle(T)$ [9]

$$
\begin{array}{r}
\frac{\Sigma(T)}{\Sigma}=1-\frac{3 M_{\pi} T}{4 \pi^{2} F_{\pi}^{2}} \sum_{n=1}^{\infty} \frac{1}{n} K_{1}\left(n \frac{M_{\pi}}{T}\right) . \\
\frac{\left\langle G^{2}\right\rangle(T)}{\left\langle G^{2}\right\rangle}=1-\frac{24}{b} \frac{M_{\pi}^{3} T}{\left\langle G^{2}\right\rangle} \sum_{n=1}^{\infty} \frac{1}{n} K_{1}\left(n \frac{M_{\pi}}{T}\right) .
\end{array}
$$

Analytic temperature dependence of the quark condensate (28) perfectly agrees, in the low temperature region, $T \lesssim M_{\pi}$, with the numerical calculations of $\langle\bar{q} q\rangle(T)$ obtained at the three-loop level of the chiral perturbation theory (ChPT) with non-zero quark mass [12. The gluon condensate slightly varies with the increase of the temperature $\left(\Delta\left\langle G^{2}\right\rangle /\left\langle G^{2}\right\rangle \sim 10^{-3}\right.$ at $T=M_{\pi}$ and $\left.\left\langle G^{2}\right\rangle=0.5 \mathrm{GeV}^{4}\right)$, i.e. the situation is similar to the ChPT [13].

Turning back, the relation (28,29) can be easily verified via direct calculation using (31), (32) and the GMOR relation. Thus in the low temperature region when the excitations of massive hadrons and interactions of pions can be neglected, equation (29) becomes a rigorous QCD theorem.

As it was mentioned above the pion plays an exceptional role in thermodynamics of QCD due to the fact that its mass is numerically close to the phase transition temperature while the masses of heavier hadrons are several times larger than $T_{c}$. This was the reason we did not consider the role of massive states in the low temperature phase. This question was discussed in detail in Ref. 12. It was shown there that at low temperatures, the contribution to $\langle\bar{q} q\rangle$ generated by the massive states is very small, less than $5 \%$ if $T$ is below $100 \mathrm{MeV}$. At $T=150 \mathrm{MeV}$, this contribution is of the order of $10 \%$. The influence of thermal excitations of massive hadrons on the properties of the gluon and quark condensates in the framework of the conformal-nonlinear $\sigma$ - model was also studied in detail in 114.

4. It was shown that the temperature derivatives of the anomalous and normal (quark massive term) contributions to the trace of the energy-momentum tensor in QCD with light quarks are equal to each other in the low temperature region.

Let us consider some physical consequences and possible applications of this relation. To this end we introduce the function

$$
\delta_{\theta}(T)=\frac{\partial}{\partial T}\left\langle\theta_{\mu \mu}^{g}-\theta_{\mu \mu}^{q}\right\rangle
$$

As it was stated above, the function $\delta_{\theta}(T)$ at low temperatures is, with good accuracy, close to zero. In the vicinity and at the phase transition point, i.e. in the region of nonperturbative vacuum reconstruction this function 
changes drastically. To see it, we first consider pure gluodynamics. It was shown in 15 using the effective dilaton Lagrangian, that gluon condensate decreases very weakly with the increase of temperature, up to phase transition point. This result is physically transparent and is the consequence of Boltzmann suppression of thermal glueball excitations in the confining phase.

Further, in Refs. [16] the dynamical picture of deconfinement was suggested based on the reconstruction of the nonperturbative gluonic vacuum. Namely, confining and deconfining phases according to [16] differ first of all in the vacuum fields, i.e., in the value of the gluon condensate and in the gluonic field correlators. It was argued in [16] that color-magnetic (CM) correlators and their contribution to the condensate are kept intact across the temperature phase transition, while the confining colorelectric (CE) part abruptly disappears above $T_{c}$. Furthermore, there exist numerical lattice measurements of field correlators near the critical transition temperature $T_{c}$, made by the Pisa group [17], where both CE and CM correlators are found with good accuracy. These data clearly demonstrate the strong suppression of CE compo- nent above $T_{c}$ and persistence of CM components. Thus, the function $\delta_{\theta}(T)_{G D}=\partial\left\langle\theta_{\mu \mu}^{g}\right\rangle / \partial T$ can be presented as a $\delta$-function smeared around the critical point $T_{c}$ with the width $\sim \Delta T$ which defines the fluctuation region of phase transition.

Similar, but more complicated and interesting situation takes place in the theory with quarks. The function $\delta_{\theta}(T)$ contains the quark term, proportional to the chiral phase transition order parameter $\langle\bar{q} q\rangle(T)$. So it is interesting to check the relation (29) and to study the behavior of the function $\delta_{\theta}(T)$ in the lattice QCD at finite temperature. It would allow both to test the nonperturbative QCD vacuum at the low temperatures in the confining phase and to extract additional information on the thermal phase transitions in QCD.

\section{ACKNOWLEDGMENTS}

I am grateful to Yu.A.Simonov for useful discussions and comments. The financial support of RFFI grant 0002-17836 and INTAS grant CALL 2000 N 110 is gratefully acknowledged.
[1] F. E. Low, Phys.Rev. 110, 974 (1958).

[2] V. A. Novikov, M. A. Shifman, A. I. Vainshtein and V. I. Zakharov Nucl. Phys. B191, 301 (1981); Sov. J. Part. Nucl. 13, 224 (1982); A. A. Migdal and M. A. Shifman, Phys. Lett. B114, 445 (1982).

[3] P. J. Ellis, J. I. Kapusta and H.-B. Tang, Phys. Lett. B443, 63 (1998).

[4] I. A. Shushpanov, P. J. Ellis and J. I. Kapusta, Phys. Rev. C59, 2931 (1999).

[5] N. O. Agasian and I. A. Shushpanov, JETP Lett. 70, 717 (1999); Phys. Lett. B472, 143 (2000).

[6] N. O. Agasian, Phys. Lett. B488, 39 (2000); Yad. Fiz. 64, 608 (2001).

[7] The deconfining phase transition temperature is the one obtained in lattice calculations $T_{c}\left(N_{f}=2\right) \simeq 173 \mathrm{MeV}$ and $T_{c}\left(N_{f}=3\right) \simeq 154 \mathrm{MeV}$. F. Karsch, E. Laermann and A. Peikert,Quark Mass and Flavour Dependence of the QCD Phase Transition, hep-lat/0012023: F. Karsch, Lattice Results on QCD Thermodynamics, hep-ph/0103314.

[8] At zero quark mass the gas of massless noninteracting pions is obviously scale-invariant and therefore does not contribute to the trace of the energy-momentum tensor and correspondingly to the gluon condensate $\left\langle\left(G_{\mu \nu}^{a}\right)^{2}\right\rangle$.

[9] N. O. Agasian, Nonperturbative vacuum and condensates in QCD below thermal phase transition, hep-ph/0104014.
[10] T. Muta, Foundations of Chromodynamics, World Scientific Lecture Notes in Physics 57 (World Scientific, Singapore, 1998).

[11] The equation (18) contains corrections $m_{q} \partial\langle\bar{q} q\rangle / \partial m_{q} \sim O\left(m_{q}\right)$ which are negligible for $u$ and $d$ quarks.

[12] P. Gerber and H. Leutwyler, Nucl. Phys. B321, 387 (1989).

[13] H. Leutwyler, Restoration of Chiral Symmetry, Lecture given at Workshop on Effective Field Theories, Dobogoko, Hungary, 1991; Bern Univ. -BUTP-91-43.

[14] N. O. Agasian, D. Ebert and E.-M. Ilgenfritz, Nucl. Phys. A637, 135 (1998).

[15] N. O. Agasian, JETP Lett. 57, 208 (1993).

[16] Yu. A. Simonov, JETP Lett. 54, 256 (1991); 55, 605 (1992); Phys. Atom. Nucl. 58, 309 (1995); "Hot nonperturbative QCD", Lecture at the International School of Physics "Enrico Fermi", Varena, June 1995, hep-ph/9509404: Analytic Methods in Nonperturbative QCD, hep-ph/0011114.

[17] A. Di Giacomo, E. Meggiolaro and H. Panagopoulos, Nucl. Phys. Proc.Suppl. 54A, 343 (1997), heplat/9608008; Nucl. Phys. B483, 371 (1997); 